\title{
A simple synthesis of mesoporous carbons with tunable mesopores using a colloidal template-mediated vapor deposition polymerization
}

\author{
Jyongsik Jang, * Byungkwon Lim and Moonjung Choi \\ Received (in Cambridge, UK) 6th May 2005, Accepted 29th June 2005 \\ First published as an Advance Article on the web 22nd July 2005 \\ DOI: $10.1039 / \mathrm{b506265a}$
}

Mesoporous carbons with highly uniform and tunable mesopores were fabricated by one-step vapor deposition polymerization (VDP) using colloidal silica particles as templates and polyacrylonitrile (PAN) as a carbon precursor.

Mesoporous carbons are used as catalysts, adsorbents and various supports. In particular, mesoporous carbons with narrow pore size distributions and tunable pore sizes are important in the field of size-selective separation, and can be applied as packing materials in chromatography or size-selective adsorbents. Recently, templating approaches have been extensively studied for the synthesis of mesoporous carbons using mesoporous and colloidal silicas as templates. Mesoporous silicas are representative porous silica templates. ${ }^{1}$ Ryoo et al. reported the synthesis of ordered mesoporous carbons (CMKs) with high surface areas and large pore volumes from ordered mesoporous silica templates. ${ }^{2}$

Compared to porous silica templating, the use of commercially available colloidal silicas as templates provides a more convenient way to fabricate mesoporous solids including carbons. ${ }^{3}$ It does not require the pre-synthesis of suitable porous silica templates at a high cost and by a complicated synthetic procedure. Moreover, the pore diameter of the mesoporous carbon can be easily controlled using an appropriate colloidal silica, which is available in a wide range of particle sizes. Mallouk and co-workers synthesized mesoporous polymers with tunable pore sizes from colloidal silica templates. ${ }^{4}$ Jaroniec and co-workers reported the synthesis of mesoporous carbons by a colloidal imprinting approach. ${ }^{5}$ In colloidal silica templating, however, it is likely that silica aggregates composed of a few particles rather than a single silica particle act as templates for the formation of mesopores. ${ }^{6}$ This leads to undesirable broadening of the pore size distribution in the resulting mesoporous carbon. ${ }^{7}$

To date, impregnation of colloidal silica templates with carbon precursors has commonly depended on the wet chemical method, where the carbon precursor in the liquid state is inserted into the templates. Recently, our group reported that the vapor deposition polymerization (VDP) technique allows a simple route for the synthesis of inorganic-polymer and inorganic-carbon core-shell nanostructures by consecutive polymerization of vaporized monomer under vacuum onto the desired surface. ${ }^{8}$ So far, VDP has been mainly performed to fabricate various thin films and core-shell nanostructures, ${ }^{9}$ whereas considerably less attention has been paid to the application of VDP in colloidal templating. The

Hyperstructured Organic Materials Research Center and School of Chemical Engineering, College of Engineering, Seoul National

University, Shinlimdong 56-1, Seoul, 151-742, Korea.

E-mail: jsjang@plaza.snu.ac.kr; Fax: +82 2888 1604; Tel: +82 28807069
VDP process makes it possible for monomer vapor to be polymerized even inside very small void spaces between colloidal particles without disrupting the characteristic of the colloidal template structure. In addition, it provides experimental simplicity such as a solvent-free system and no recovery process. In this communication, we report a simple route for the synthesis of mesoporous carbons with highly uniform and tunable mesopores using colloidal silica templating and polyacrylonitrile (PAN), synthesized by the VDP method, as a carbon precursor.

Scheme 1 illustrates the overall synthetic procedure for forming mesoporous carbons. Ludox SM-30, HS-40, and TM-40 aqueous colloidal solutions were used as sources of silica particles with diameters of 7, 12, and $22 \mathrm{~nm}$, respectively. In a typical synthesis of mesoporous carbons, $0.2 \mathrm{~mL}$ of chlorodimethylvinylsilane (CDVS) was added to $40 \mathrm{~mL}$ of distilled water containing $1 \mathrm{~mL}$ of colloidal silica solution. The CDVS treatment imparts hydrophobicity to the silica particle surfaces allowing for the better adsorption of monomer vapor on the silica templates. ${ }^{10}$ The mixture solution was stirred overnight and then dried to obtain a powder form of the colloidal silica templates. The transmission electron microscopy (TEM) image of the CDVS-treated silica particles dried from water showed that the particles were nearly monodisperse and existed as single particles (Fig. 1a). By contrast, the silica particles prepared without CDVS treatment formed a large agglomerate after drying (Fig. 1b). These results mean that

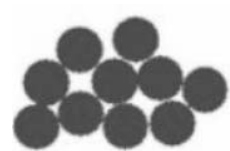

Silica colloids

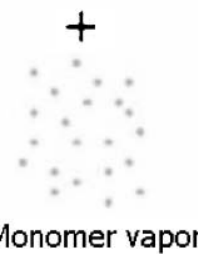

Monomer vapor
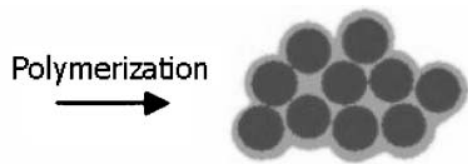

Silica-PAN nanocomposite

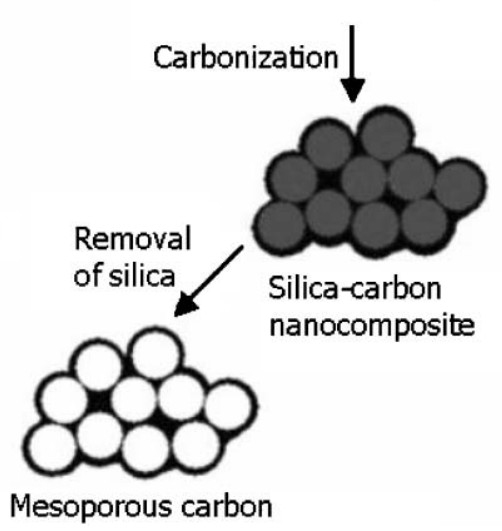

Scheme 1 Schematic illustration for the synthesis of mesoporous carbons. 
(a)

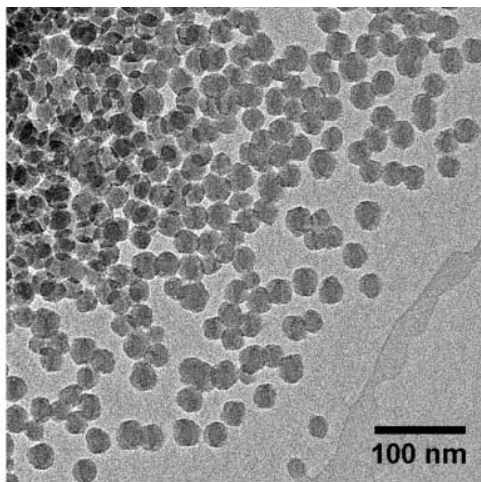

(b)

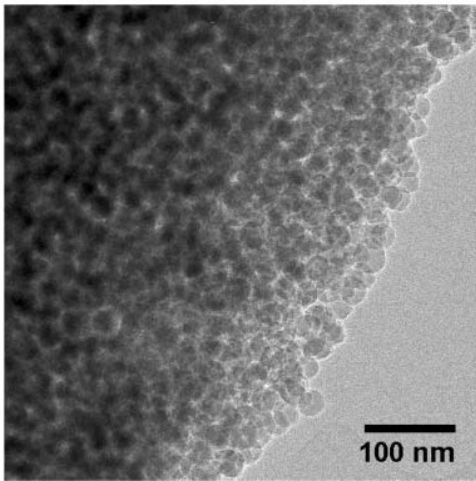

Fig. 1 TEM images of (a) CDVS-treated and (b) untreated silica particles after drying from water. An average size of silica particles is $22 \mathrm{~nm}$.

CDVS treatment of the silica particles plays an important role in obtaining single silica particles after the drying process. It might be due to the fact that the silica particles, whose surfaces are covered by organic groups such as methyl and vinyl groups from the CDVS treatment in this experiment, are not likely to form siloxane bonding between silica particles, and consequently a large agglomerate as well.

To obtain silica-PAN nanocomposites, $0.5 \mathrm{~g}$ of CDVS-treated silica particles and $0.01 \mathrm{~g}$ of a free radical initiator, benzoyl peroxide (BPO), were added to the reactor, and then the reactor was evacuated to $c a .10^{-1}$ Torr at room temperature. $0.5 \mathrm{~mL}$ of acrylonitrile monomer was introduced into the reactor and vaporized at an elevated temperature of $90{ }^{\circ} \mathrm{C}$. The vaporized acrylonitrile was incorporated into the void spaces of the colloidal silica templates and polymerized with an initiation by BPO. The VDP reaction proceeded for $24 \mathrm{~h}$ and the silica-PAN nanocomposite was obtained. The FT-IR analysis of the silica-PAN nanocomposite exhibited the characteristic PAN peaks of $\mathrm{C}-\mathrm{H}$ stretching vibration at $2900 \mathrm{~cm}^{-1}, \mathrm{C} \equiv \mathrm{N}$ stretching vibration at $2250 \mathrm{~cm}^{-1}$, and $\mathrm{C}-\mathrm{H}$ bending vibration at $1450 \mathrm{~cm}^{-1}$, which confirmed the successful polymerization of acrylonitrile.

The carbonization of the silica-PAN nanocomposite was performed at $900{ }^{\circ} \mathrm{C}$ for $5 \mathrm{~h}$ under $\mathrm{N}_{2}$ flow and subsequent dissolution of the silica template using HF solution produced the mesoporous carbons. These PAN-based mesoporous carbons (PMCs) prepared using 7, 12, and $22 \mathrm{~nm}$ sized silica particles as templates, were designated as PMC-7, PMC-12, and PMC-22, respectively. Fig. 2 shows the TEM images of the PMCs with different pore diameters. It revealed that all the PMCs had the typical morphology of mesoporous materials with a 3-dimensionlly (a)

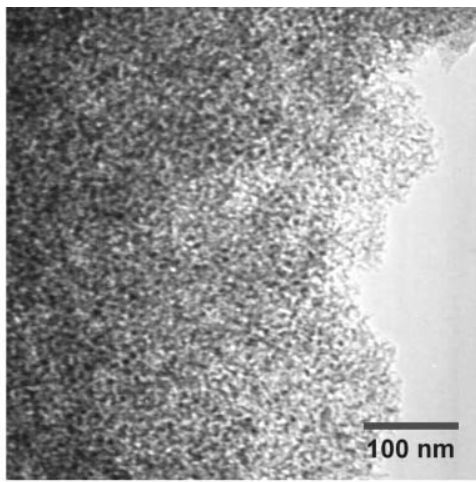

(b)

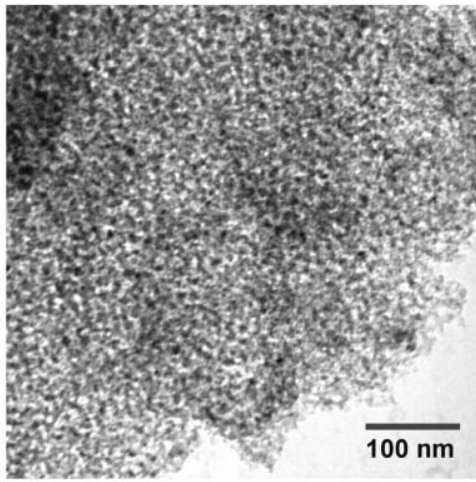

(c)

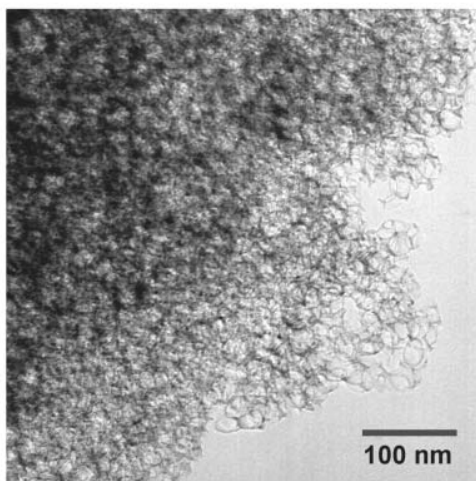

Fig. 2 TEM images of the mesoporous carbons prepared using (a) 7, (b) 12, and (c) $22 \mathrm{~nm}$ sized silica particles as templates.

interconnected and disordered pore structure. The mesopores are evident in carbon frameworks and entirely close-packed. It demonstrates that the mesopores in the carbons were generated by removal of the silica particles and remained intact during the etching process. In the TEM image of PMC-22, spherically shaped mesopores are clearly shown.

The pore structures of the PMCs were analyzed by nitrogen adsorption-desorption isotherms at $77 \mathrm{~K}$. All the isotherms of the PMCs showed large hysteresis loops with sharp adsorption and desorption branches at high relative pressures corresponding to type IV behavior, indicating the presence of mesopores (Fig. 3a). The sharpness of the isotherm indicates the narrow pore size distribution (PSD). The PSD curves calculated from BarrettJoyner-Halenda (BJH) method from nitrogen adsorption branches exhibited very narrow pore size distributions centered at 7.4, 12.1, and $22.0 \mathrm{~nm}$ for PMC-7, PMC-12, and PMC-22, respectively (Fig. 3b). These values are highly consistent with the sizes of single silica particles used as templates in each case. It 


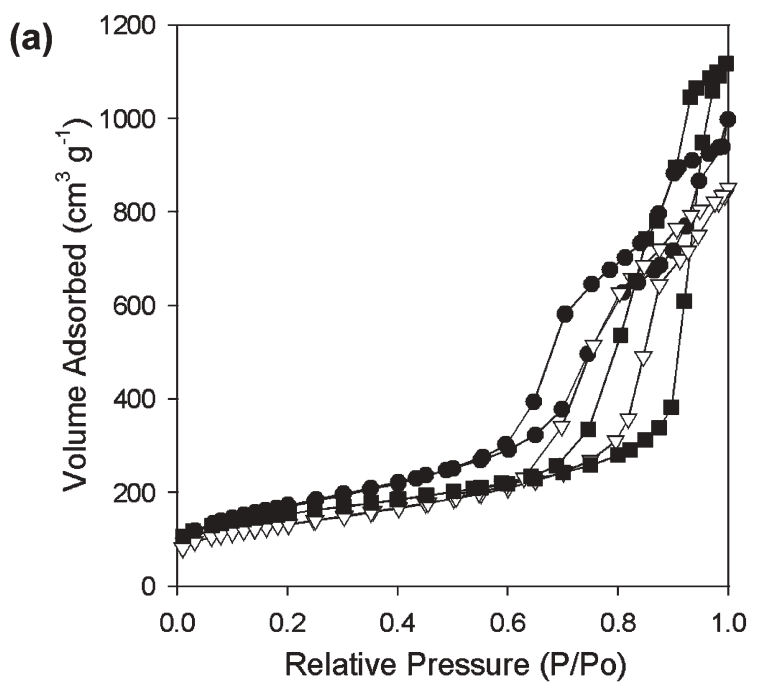

(b)

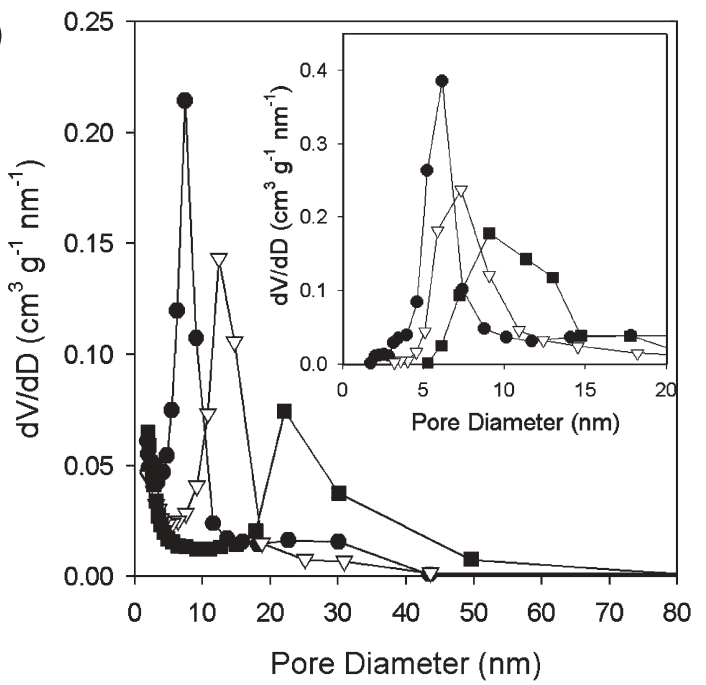

Fig. 3 (a) Nitrogen adsorption-desorption isotherms of the PMCs and (b) their corresponding pore size distribution (PSD) curves derived from the adsorption and desorption (inset) branches (PMC-7, •; PMC-12, $\nabla$; PMC-22, 口).

demonstrates that a single silica particle acted as a real template for the generation of mesopores in the carbon. In addition, the narrow pore size distribution corresponding to the size of the silica template particle confirmed the feasibility of our approach for easy control over the pore diameter of the mesoporous carbon.

The pore size analysis derived from the desorption branches of the isotherms also showed relatively narrow pore size distributions centered at 6.1, 7.3, and 9.1 nm for PMC-7, PMC-12, and PMC22, respectively (Fig. 3b, inset). These pores correspond to the windows that interconnect the main cell pores, which indicates that the pore system of the PMCs is an ink-bottle-like structure composed of the main cell pores interconnected by uniform windows. ${ }^{11}$ In a previous report on the fabrication of mesoporous polymers from colloidal silica templates, the silica particles were sintered at an elevated temperature in order to provide pore connectivity in the mesoporous polymer replicas. ${ }^{4}$ In this approach, however, the PMCs exhibited excellent pore connectivity without exhaustive heat treatment for sintering of silica particles. For PMC-7, PMC-12, and PMC-22, the BrunauerEmmett-Teller (BET) surface areas were as high as 612, 473 and $542 \mathrm{~m}^{2} \mathrm{~g}^{-1}$, and the total pore volumes were $1.52,1.29$ and $1.72 \mathrm{~cm}^{3} \mathrm{~g}^{-1}$, respectively.

In summary, a VDP technique combined with colloidal silica templating is found to be a simple and effective method to fabricate mesoporous carbons with tunable pore diameters. The mesoporous carbons provided the narrow pore size distributions as well as excellent pore connectivity. This novel approach might be expanded to allow the preparation of various mesoporous structures composed of polymers or carbons.

This work was supported by the Brain Korea 21 program of the Korea Ministry of Education and Korea Science and Engineering Foundation through the Hyperstructured Organic Materials Research Center.

\section{Notes and references}

1 J. Lee, S. Yoon, S. M. Oh, C.-H. Shin and T. Hyeon, Adv. Mater., 2000, 12, 359; T. Kyotani, T. Nagai, S. Inoue and A. Tomita, Chem. Mater., 1997, 9, 609; Z. Ma, T. Kyotani and A. Tomita, Chem. Commun., 2000, 2365; D. Kawashima, T. Aihara, Y. Kobayashi, T. Kyotani and A. Tomita, Chem. Mater., 2000, 12, 3397; A. Lu, A. Kiefer, W. Schmidt and F. Schuth, Chem. Mater., 2004, 16, 100.

2 S. H. Joo, S. J. Choi, I. Oh, J. Kwak, Z. Liu, O. Terasaki and R. Ryoo, Nature, 2001, 412, 169; S. Jun, S. H. Joo, R. Ryoo, M. Kruk, M. Jaroniec, Z. Liu, T. Ohsuna and O. Terasaki, J. Am. Chem. Soc., 2000, 122, 10712; J. S. Lee, S. H. Joo and R. Ryoo, J. Am. Chem. Soc., 2002, 124, 1156; R. Ryoo, S. H. Joo and S. Jun, J. Phys. Chem. B, 1999, 103, 7743 .

3 A. A. Zakhidov, R. H. Baughman, Z. Iqbal, C. Cui, I. Khayrullin, S. O. Dantas, J. Marti and V. G. Ralchenko, Science, 1998, 282, 897; J. Jang and B. Lim, Adv. Mater., 2002, 14, 1390; F. Schuth, Angew. Chem., Int. Ed., 2003, 42, 3604; G. J. de A. A. Soler-Illia, C. Sanchez, B. Lebeau and J. Patarin, Chem. Rev., 2002, 102, 4093; S. Polarz and B. Smarsly, J. Nanosci. Nanotechnol., 2002, 2, 581.

4 S. A. Johnson, P. J. Ollivier and T. E. Mallouk, Science, 1999, 283, 963.

5 Z. Li and M. Jaroniec, J. Am. Chem. Soc., 2001, 123, 9208.

6 S. Han and T. Hyeon, Carbon, 1999, 37, 1645.

7 S. Han and T. Hyeon, Chem. Commun., 1999, 1955.

8 J. Jang and B. Lim, Angew. Chem., Int. Ed., 2003, 42, 5600

9 J. Bai, C. M. Snively, W. N. Deglass and J. Lauterbach, Macromolecules, 2001, 34, 1214; J. Bai, C. M. Snively, W. N. Deglass and J. Lauterbach, Adv. Mater., 2002, 14, 1546.

10 H. T. Oyama, R. Sprycha, Y. Xie, R. E. Partch and E. Matijevic, J. Colloid Interface Sci., 1993, 160, 298.

11 J. Lee, K. Sohn and T. Hyeon, J. Am. Chem. Soc., 2001, 123, 5146. 